\title{
Investigation of Channel Coding Techniques for High Data Rate Mobile Wireless Systems
}

\author{
Samridhi \\ ECE Department \\ Guru Nanak Dev University, \\ Regional Campus, Jalandhar (India)
}

\author{
Jyoteesh Malhotra, Ph.D. \\ ECE Department \\ Guru Nanak Dev University, \\ Regional Campus, Jalandhar (India)
}

\begin{abstract}
$21^{\text {st }}$ century certainly belongs to a new wireless world. Be it voice or data communication, in every walk of life wireless is the call in this era. The need of the present scenario for wireless applications is the enhanced data rate for various applications of communication along with reduction in power requirements. But during message transferal, the data might get corrupted due to impairments of channels, affecting both the needs of data rate as well as power. Reduction of error due to noisy channels becomes critical. Here, channel coding comes out as bliss. These techniques are used for rendering reliable information through the transmission channel to the user. These approaches preserve data while it is being transported through channel. When error occurs, channel coding detects and corrects it and helps the communication systems design to reduce the noise effect during transmission. It is due to endowment of these coding techniques that we are today capable to reach near Shannon limits. The purpose of this paper is to study and analyze the performance and efficiency of different Forward error correcting (FEC) codes. Their improvement in performance is compared to tradeoffs in complexity and decoding lag. It has been observed that though selection of code is dependent on in-hand application but turbo codes outrun other codes in many aspects.
\end{abstract}

Keywords

Turbo, Convolution, LDPC, RS codes.

\section{INTRODUCTION}

The field of wireless and mobile communication has a phenomenal history that spans over a century of technological transformations. Wireless communication that provides voice only is not adequate to support the requisites of user. With the outbreak in mobile telephone, Internet and multimedia services, wireless has become one of the most prevalent core technology enablers for distinctive computing and communications applications. But multipath fading and several types of interferences limit the performance of wireless system. It is very important to enhance quality of service (QoS) as well as diminish time and resource consumption in wireless communication networks by lowering the losing probability of data packets.

A channel characterized by Gaussian noise defines a limit, about the maximum channel capacity and error free transmission rate for a given signal-to-noise ratio (SNR) and channel bandwidth, known as Shannon's Limit. FEC codes yield algorithms for encoding and decoding data bits, and helps in reaching data rates closer to Shannon's Limit by combating all the problems. For data transmission with higher bit error rate, wireless systems needs implementation of various such coding techniques.
Channel coding is a method of encoding data in a communication channel that affixes patterns of redundancy into the transmission path so as to lower the error rate. In a controlled manner, number of symbols in the source encoded message is increased so as to facilitate two basic objectives of error detection and error correction at receiver end. The amount of error detection and correction required and its efficiency is dependent on SNR. The prominent edge of forward error correction is that a back-channel is not required and retransmission of data can usually be avoided but at the expense of higher bandwidth requirements on average.

Redundancy is added to data in FECs in the form of parity bits. In general, more the generation of parity pits per data bit, more the potential errors can be detected and corrected. Different FECs avail different methods to check and correct errors in an attempt to close in on the limit suggested by Shannon for data transmission. Here, BCH codes, ReedSolomon codes, LDPC, STBC, convolution and turbo codes are examined, and their dissimilarities are briefly discussed, along with the pros and cons of each approach.

The paper is organized as follows: Section 2 introduces the necessary concepts about different coding techniques. Section 3 includes the literature survey explaining the status of analyzed coding schemes in wireless set-ups. Section 4 explains the associating issues of coding schemes. Section 5 proposes future scope and open issues associated with mentioned channel coding approaches. Finally last section concludes this paper.

\section{TAXONOMY OF CODING}

Channel codes can be stratified broadly in two categories: block codes and convolution codes. For real-time errorcorrection, convolution codes are preferred. Smaller codeword usage in convolution codes can achieve the same quality as obtained with longer code-words in block coding. In block codes, $\mathrm{n}$ bit code-word can be obtained by encoding a block of $\mathrm{k}$ information bits. Whereas in convolution coding, encoded $\mathrm{n}$ bit sequence depends on previous information bits too along with current information bits [8]. It means convolution codes have memory requirement and block codes are memory less. Diverse categories of both block and convolution codes have been stated as:

\subsection{Low Density Parity Check (LDPC)}

LDPC codes are class of forward error-correction codes, first presented in 1962. At that time, their incredible capabilities remained undiscovered because of the composite computational demands of simulation. In mid 90s these codes came into limelight again with new generalizations. LDPC codes are fundamentally linear codes acquired from sparse bipartite graphs. These are block codes with parity-check matrices having very smaller number of non-zero entries. It is 
the sparseness of $\mathrm{H}$ which results in both the decoding complexity and a minimum distance.

The biggest difference between LDPC codes and classical block codes is the way in which they are decoded. Maximum Likelihood (ML) like algorithms are preferred for classical decoding block codes which are usually shorter and are designed algebraically to make this task of decoding less complicated. On the other hand, LDPC codes are decoded iteratively with the help of graphical representation of their parity-check matrix.

Message-passing algorithm is the class of decoding algorithms used to decode LDPC [12]. Their working can be defined by the flow of messages along the edges of a Tanner graph. Each Tanner graph node has access only to the information accommodated in the messages on the edges connected to it. It works in isolation. The messages proceed back and forward between the bits and check the nodes iteratively until desired outcome is achieved (or the process halted). In some algorithms, like in bit-flipping decoding, the messages are in binary form and in others methods, such as belief propagation decoding, the messages are in the form of probabilities representing a level of belief about the value of the code-word bits.

LDPCs are advantageous due to many reasons; parallelizable decoder, lower error rate floor, superior performance in bursts; no requirement of interleavers. It has been utilized in diverse applications like deep space and satellite communication, magnetic storage, internet packet transferal. These codes can approach Shannon Limit but at the cost of very high complexities as compared to other simplified codes like turbo codes.

\subsection{Reed-Solomon (RS) Coding}

The acronym BCH comprises the initials of its inventor's names Alexis Hocquenghem, Raj Bose and D. K. RayChaudhuri. It is one of the most efficient linear block coding techniques. For single bit error correction $\mathrm{BCH}$ performance is similar to Hamming code. Applying low power $\mathrm{H} / \mathrm{W}$ its simplified syndrome decoder can be easily constructed. It gives this coding an edge.

RS codes can be considered as non-binary $\mathrm{BCH}$ codes. RS codes are $\mathrm{BCH}$ codes where values of the code coefficient are taken from Galois Field GF $\left(2^{\mathrm{m}}\right)$ [2].

It is an error-correcting coding system that was formulated to handle the issue of correcting multiple errors [3]. It is preferred especially in mass storage devices (hard disk drives, DVD, barcode tags), both wireless and mobile communications units, satellite links, digital TV, digital video broadcasting (DVB), and modem technologies like xDSL [2][3].

With the addition of $\mathrm{t}$ check symbols to the data, detection of any combination of up-to t erroneous symbols and correction up to [t/2] symbols can be made by RS codes. These codes differentiate from a Hamming code in the fact that it encodes groups of bits rather than encoding one bit at a time.

Syndromes are calculated for each code-word so as to correct or erase errors by RS decoder. Based upon the syndromes the decoder can determine the number of errors in the received block. After finding that error has occurred, the decoder tries to find the locations of the errors using the Berlekamp-massey algorithm with the generation of an error locator polynomial. The roots of this polynomial are obtained using the Chien search algorithm. Using Forney's algorithm, the symbol error values are found and corrected.

\subsection{Space Time Codes (STC)}

Scope of transmit diversity for the coherent multiple-antenna flat-fading channel ambits from space-time block codes (STBC) to space-time trellis codes (STTC). These are two main types of STCs. Space-time block codes work on a block of input symbols, generating a matrix output over antennas and time [4]. Space-time trellis codes work on one input symbol at a time giving a sequence of spatial vector outputs.

By concatenating STBC with fundamental AWGN trellis codes for lesser number of receive antennas and trellis states, STBCs can efficiently surpass space-time trellis codes. Capacity loss incurred by STBC is the prominent reasons of performance loss for higher number of receive antennas.

For single-antenna channel, just like tradition trellis coded modulation (TCM), STTCs deliver coding gain. With the combined ability to furnish full diversity gain and coding gain, STTCs have inherent advantages over STBCs. For higher number of trellis states and multiple receive antennas STTCs outshine STBCs but at the cost of very expensive, difficult to design as well as complex encoder/decoders. STBCs are proficient in delivering a diversity order equal to the product of the number of receive and transmit antennas.

Tarokh, Jafarkhani, and Calderbank proposed orthogonal designs applicable to space-time block codes for wireless applications and generalized the Alamouti scheme for more than two transmit antennas [5]. This is a very special type that can attain its full diversity gain without any need to sacrifice its data rate. An exceptional link between orthogonal designs and wireless communications is furnished by these codes. Unlike traditional single-antenna AWGN block codes, coding gain is missed by full rate space-time block codes. Their salient feature is the provision of full diversity with exceptionally low encoder/decoder complexity. STBCs can be divided into two main classes, namely,

1. Orthogonal Space-Time Block Codes (OSTBCs) and

2. Non-Orthogonal Space-Time Block Codes (NOSTBCs).

The Quasi-Orthogonal Space-Time Block Codes (QSTBCs) belong to class of NOSTBCs. The OSTBCs attain full diversity with low decoding complexity, but at the expense of some loss in data rate. For more than two transmit antennas full data rate can be secured with QSTBCs with a small loss of the diversity gain. Moreover, it has been exhibited that QSTBCs can be conducted even better than OSTBCs in the SNR range of practical interest (up to $20 \mathrm{~dB}$ ).

\subsection{Convolution Coding}

The distinction between block codes and convolutional codes is their encoding principles. In the block codes, the information bits proceeded by the parity bits. In convolutional codes, the information bits are spreaded along the sequence. That means, the convolutional codes map information in code bits and not block wise. These codes sequentially convolve the sequence of information bits according to some rule. The convolutional code is linear.

With the combination of various subsets of bits in the window, the encoder utilizes a sliding window for calculation of parity bits. The combining is a simple addition (mod- 2 or exclusive or). Unlike a block code, however, the windows 
overlap and slide by 1 . The depth of the window, in bits, is called the code's constraint length.

The longer constraint lengths result in more number of parity bits that are affected by any given message bit. Due to the fact that the parity bits are the only bits transmitted over the channel, a larger constraint length generally implies a greater toughness to bit errors. The trade-off, though, is that it will take considerably more time to decode codes of long constraint length. The complexity of decoding increases exponentially in the constraint length, so one cannot increase the constraint length arbitrarily and expect fast decoding. Secondly, greater value of rate means higher resilience of bit errors, but the trade-off here is that a proportionally higher requirement of communication bandwidth that is devoted to coding overhead.

The receiver should decide the "best possible" sequence of transmitter states. There are many way- outs to define "best", but one that is especially fascinating is the most likely sequence of states (i.e., message bits) that the transmitter must have been traversed (sent). The maximum-likelihood (ML) decoder can be used for the convolutional code.

Another approach that overcomes its shortcomings is Viterbi decoding. This decoding method uses a special structure called the trellis [9]. The state machine view represents what happens at each instant when the sender has a message bit to process, but doesn't represents how the system evolves in time. It is the trellis structure that makes the time evolution explicit. Decoder gets a sequence of parity bits, and needs to determine the best possible path through the trellis.

\subsection{Turbo Coding}

The significance of turbo codes is that they make sure the reliable communications with power efficiencies closer to the theoretical limit predicted by Claude Shannon. Some benefits of using Turbo codes are that they efficiently outperform conventional codes and they utilize interleavers diminishing burst errors. Some of the magnificent performance characteristics of Turbo codes include better coding gain compared with an un-coded channel and more than $2.7 \mathrm{~dB}$ better coding gain comparatively conventional Viterbi/Reed Solomon. Such coding power is extremely significant in many telecommunications applications because of its impact on either increase in signal range and decrease in transmission power of signal. Such coding gain also enhances battery life for portable components and for multiple-access or a cellular scenario, cell capacity is increased as well. In a simplified turbo code, there are two convolutional encoders in parallel.

The code-word in this coding scheme consists of the input bits - i.e. the code is systematic - proceeded by the parity check bits from the first encoder and then different parity bits from the second encoder. In general, one can apply multiple turbo encoders having more than two branches. The convolutional code at each branch is called the constituent code (CC). Generator functions can be same or different for various CCs. Both the transfer function and the distance spectrum are very useful in developing a performance bound for a given code. In turbo codes Recursive Systematic Convolutional (RSC) codes are demonstrated to perform better than the non-recursive ones. The trellis and the free distance $\left(\mathrm{d}_{\text {free }}\right)$ will be similar for both codes. Puncturing can be introduced too to increase the rate of the convolutional code beyond that resulting from the basic composition of the encoder.

Interleaving is utilized to feed the encoders with permutations so that the generated redundancy sequences can be assumed self-sufficient. Another key role of the interleaver is to shape the weight distribution of the code that ultimately controls its performance.

The decoder results in an iterative working. Practically, the number of iterations does not exceed 18, and in many cases 6 iterations can produce satisfactory performance [16]. Actually, the term turbo code is given because of this iterative decoder scheme with reference to the turbo engine principle. The first decoder will decode the sequence and then send the hard decision along with a reliability estimation of this decision to the next decoder. Now, the second decoder has an advantage of having an extra information for the decoding; a priori value together with the sequence.

Algorithms applied for decoding convolutional codes can be modified so as to be applied in decoding turbo codes. Viterbi algorithm is an optimal decoding method that diminishes the probability of sequence error for convolutional codes. A modified version of Viterbi algorithm, called SOVA (Soft Output Viterbi Algorithm), uses soft outputs can be used in turbo codes. Iterative SOVA output approaches maximum likelihood (ML) decoding performance bound simply with increase in the number of iterations. However, the question of convergence is still waiting for a concrete answer.

\section{LITERATURE SURVEY}

Telagarapu in [1] presented the usage of forward error correction techniques in WiMax standard. The supremacy of such techniques is the elimination of back-channel. These are required so as to enhance the reliability of communication over noisy channels. Analysis has been done using ReedSolomon coding and convolution coding scheme, cyclic prefix and interleaving for various modulation techniques with respect to bit-error rate and SNR ratio. Long sequences of ones and zeros in each burst of input data can be avoided using scrambling. After this, redundant bits are added to data blocks according to some prescribed algorithm. Using ReedSolomon technique, decoder can correct burst errors, reduces bit-error rate and hence delivers error-free data to subscriber.

Shrivastava in [2] analyzed one of the most robust known classes of linear cyclic block codes which are also subset of $\mathrm{BCH}$ codes i.e. Reed-Solomon codes. It is explained that smaller code of any desired size from a larger code can be produced using a technique known as "shortening". For example, the widely used $(255,251)$ code can be converted to a $(160,128)$. Paper represented that different number of error bits can be observed from the plotted graph for different error capabilities. For example when $2 t=4 ; t=2$ thus, 16 error bits can be corrected at max. Corresponding range of SNR can be realized using the plotted graph. Similarly for $2 \mathrm{t}=8 ; \mathrm{t}=4$ so at max 32 bits can be repaired. This range is quite bigger the previous range of SNR. This analysis is helpful to observe that whether decoder can correct the received signal or not and this saves a lot of time and efforts.

El-Bendary in [3] explained the importance of efficient interleaver in enhancement of block codes performance over wireless channels. Moreover, using adaptive coding simplification of decoder can be expected. Paper proposed using efficient data randomize tool as an interleaver and comparing it with traditional one.

Code-rate and the length of the packet bit stream are two factors that mainly affect the performance of RS codes. BER value with the channel SNR variation using the RS $(7,3)$ and RS $(15,11)$ codes with different data streams is observed. Longer packets degrade the performance of the system. The 
amount of throughput is dependent on many factors like length of bit of packet stream, the number of retransmission times, and transmission duration. The scenarios used in the paper are applied for evaluating traditional block interleaver and the proposed chaotic interleaver compared to no interleaving encoded data transmission using RS codes. The simulation results unfolded that the block codes performance can be intensified over wireless channel using efficient interleaver.

Parveen in [4] presented the simulation results for $2 \times 2$ Alamouti Scheme. Full diversity can be obtained along with simplified linear operations at transmitter as well as at receiver. These codes are referred as Orthogonal Space-Time Block Codes (OSTBCs). Paper showed the comparisons of different schemes using BPSK modulation at constant SNR of $13 \mathrm{db}$. Better performance can be obtained using Alamouti (BER=1.1e-005) with configuration of $2 \times 2$ when compared to MRC $(B E R=0.001606)$ case. This is due to the fact that effective channel information from 2 receive antenna over 2 symbols gives diversity order of 4 . In Alamouti STBC scheme, $\left(\mathrm{H}^{\mathrm{H}} \mathrm{H}\right)$ diagonal matrix makes sure that there is no cross talk between two symbols after the equalizer and the noise term is still white.

Sethi in [5] provided the description of different types of space time block codes: Alamouti, orthogonal and quasiorthogonal. Performance analysis of these codes had been done using quasi-static Rayleigh flat fading channel. Comparison of codes is done using various modulation schemes like BPSK, QPSK, 8PSK, 16 PSK, 32PSK, 64PSK, 128PSK. Results proved that quasi-orthogonal scheme outperforms others. It is presented that using a quasiorthogonal design; pairs of transmitted symbols can be decoded individually. The application of this structure is in designing the codes yielding higher transmission rates while sacrificing the full diversity.

Manik in [6] analysed the performance of STBC-OFDM for different constraints in Rayleigh fading channels. Various effects are studied: modulation order, antenna selection techniques, slow and fast fading conditions and power conditions on the working of STBC-OFDM. Increasing the order of modulation will result in a significant SNR loss. Higher the order of modulation, higher the BER system will have. Performance comparison of equal power distributed and unequal power distributed TX antenna revealed that equal power system gives a $3 \mathrm{~dB}$ penalty when compared with unequal power system. The simulation also showed that STBC-OFDM performs significantly better with the antenna selection technique. The system works well in slow fading. If fading is somewhat rapid, performance degradation can be seen.

Kumar in [7] investigated the performance of Reed-Solomon (RS), convolutional code (CC) and concatenated (RS-CC and CC-RS) codes used for encoding in wireless communications. Both RS and CC have their own pros. On one end CC can correct random errors and on the other end RS can correct burst errors. Thus, with their collaboration optimum results can be obtained. Simulated results in paper have shown that $\mathrm{CC}$ performs better than RS. Considering the concatenated codes, CC-RS is no doubt much better than CC and RS codes but the simulation result represents clearly that RS-CC to be even better than CC-RS. Overall BER of RS-CC has significant coding gain which increases with $\mathrm{E}_{b} / \mathrm{N}_{0}$. Moreover, the slope of concatenated codes is stronger and has less flattening affect.
Jadhao in [8] analyzed the effectiveness of linear block codes, convolution codes and concatenated codes. Their underlying phenomena are given. Simulative results shown that as comparison to linear block codes and concatenated codes, convolution codes have very good capability of error correction. But they are difficult to implement too. There remains trade-offs between efficiency of channel as well as logic of encoder/decoder.

Asif in [9] presented BER (Bit Error Rate) performances for Rayleigh fading multipath channels. Using convolution coding, comparison has been made between coded and uncoded signal. Viterbi algorithm is used at receiver end. Results shown in the paper explained that when SNR is greater than $21 \mathrm{~dB}$, data encoded with convolution coding gives certainly better performance gain than un-coded data.

Gupta in [10] compared performance of irregular and regular LDPC. Concatenation has been done with Alamouti coded OFDM-MIMO systems. LDPC can be of regular or irregular type depending upon whether number of ' 1 's per row per column is fixed or varying. From simulation results, it has been shown that optimized irregular LDPC codes when concatenated with Alamouti SFBC in $2 \times 2$ MIMO-OFDM systems achieved best BER results.

Shedsale in [11] gave a review on construction methods of regular LDPC codes. It is followed by comparison of Gallager's method, Reed-Solomon and the Progressive edge growth (PEG) combinatorial method for constructing LDPC. It has been shown that when LDPC are obtained using Gallager's method shortcomings are observed; high encoding complexity for larger column weights and shorter cycles are not ensured. But better girth, minimum distance properties and low complexity can be easily obtained using other two methods.

S. Hassan in [12] investigated two very efficient and important coding schemes; turbo coding and LDPC in terms of performance and their complexity. Two rates were considered $1 / 2$ and $7 / 8$ for performance evaluation and $1 / 2,1 / 3$, $3 / 4$ and $7 / 8$ for complexity comparison. From the results it can be seen that for moderate rates turbo coding is better and for higher ones LDPC outperforms turbo. Talking about complexity, turbo codes have constant complexity independent of code rates. This is because with puncturing of basic code, different rates can be obtained. While in LDPC complexity is highly code rate dependent. Higher code rate results in lower complexity and vice-versa. Thus it can be concluded that when code rate is high, use LDPC and for lower rates go for turbo codes. Performance is more important issue so turbo codes are preferred.

Khan in [13] explained turbo and LDPC codes. Comparison has been made on the basis of certain factors. Code lengths 1784 and 3568 are used and for both of these lengths LDPC is better until a particular $\mathrm{S} / \mathrm{N}$ is reached, $\mathrm{Eb} / \mathrm{No}=1.15$ and 1.1 $\mathrm{dB}$ respectively. For complexity parameters included were number of multiplications, additions and complex operations $\left(\log , \tanh , \mathrm{e}, \tanh ^{-1}\right)$. For LDPC number of iterations may vary i.e. decoding stops as soon as desired output is obtained. Oppositely turbo code undergoes specific number of iterations each time which means time spent in decoding as well as bit rate are constant entities. Thirdly, LDPC can be implemented in parallel which is advantageous for longer codes.

Patel in [14] explained all about turbo codes; their encoding, decoding and interleaving. Performance is evaluated using different iterations. Results show that decoding performance 
improves with increase in block length. At a particular value of Eb/No, BER is less for larger block lengths. Certain block lengths are mentioned in LTE. If block length exceeds then block is subdivided into smaller portions.

Nimbalker in [15] investigated different interleavers for LTE turbo coding. The almost regular permutation (ARP) and quadratic polynomial permutation (QPP) interleavers are efficient in rectifying the problem of throughput observed in WCDMA turbo interleaver. Key features of these include flexible parallelism, proper support for Radix-4 decoding and well-organized memory. Though both the interleavers perform well but QPP was preferred for LTE. QPP has more parallelism factors, lesser interleaver parameter storage.

\section{ISSUES}

From the literature survey done in the foregoing section, it can be examined that each channel code immanent some kind of issues and challenges. These are explained as below:
Kene in [16] proposed the methods for efficient termination of iterations in decoder. Reduction in the time delay and the requirement of memory size while maintaining the BER performance were the parameters focused on. Various termination techniques like Cyclic Redundancy Check (CRC), Cross Entropy (CE) and Sign Change Ratio (SCR) are given. These approaches reduce the complexity. Results show that $\mathrm{CE}$ is the most effective of all towards very low SNR w.r.t. system stability. At pretty low SNR ranges from 0 to $2 \mathrm{~dB}$, turbo codes approaches channel capacity. All these schemes increase the speed of decoder iteration processing. Hence there is reduction in time delay without affecting system performance.

Table1. Issues and Challenges of diverse coding schemes

\begin{tabular}{|c|c|c|}
\hline Coding Scheme & & Issues and Challenges \\
\hline \multirow[t]{7}{*}{ LDPC } & i. & $\begin{array}{l}\text { Capacity approaching codes have poor error floor performances and codes with exceptionally } \\
\text { low error floors have thresholds distant from capacity. }\end{array}$ \\
\hline & ii. & $\begin{array}{l}\text { Another important practical challenge while dealing with coding scheme for adaptive air } \\
\text { interfaces is flexibility in terms of block sizes and code rates. }\end{array}$ \\
\hline & & $\begin{array}{l}\text { Better performance with a completely random LDPC can be obtained at the cost of very } \\
\text { complex interconnections. But in terms of H/W wiring, larger block lengths may not support } \\
\text { this. It further results in extremely complex encoding as well as lower achievable parallelization } \\
\text { level. }\end{array}$ \\
\hline & iii. & $\begin{array}{l}\text { Moreover Random-like LDPC suffers from problem that each code rate and block length needs } \\
\text { a new code. It further reduces flexibility. }\end{array}$ \\
\hline & iv. & $\begin{array}{l}\text { Higher memory requirement for random-like LDPC even if for encoding sparsity is maintained } \\
\text { in check matrix. }\end{array}$ \\
\hline & v. & $\begin{array}{l}\text { Introduction of min-sum approximation results in a typical performance loss of } 0.5-1 \mathrm{~dB} \text {. Loss } \\
\text { can be lowered for larger block lengths with increased iterations but with proportional } \\
\text { enhancement of decoder complexities. }\end{array}$ \\
\hline & vi. & $\begin{array}{l}\text { The classical method of message passing (called flooding) requires very high memory, together } \\
\text { with increased iterations (delay). }\end{array}$ \\
\hline \multirow[t]{6}{*}{ STBC } & i. & $\begin{array}{l}\text { Lack of coding gain. For larger number of transmit antennas their respective code designs } \\
\text { remains an open question. }\end{array}$ \\
\hline & ii. & $\begin{array}{l}\text { For increased number of transmit antennas, the shortcoming of orthogonal designs is that their } \\
\text { extant with higher rates }(>1 / 2) \text { is generally not well understood. }\end{array}$ \\
\hline & iii. & $\begin{array}{l}\text { For higher data rates and a larger number of transmit antennas, OSTBCs suffer from complexity } \\
\text { or performance shortcomings. Finding high rate }(>1 / 2) \text { OSTBCs for an arbitrary number of } \\
\text { transmit antennas is a formidable problem. }\end{array}$ \\
\hline & iv. & $\begin{array}{l}\text { In STBC designs, there is a tradeoff between information rate and decoding complexity. The } \\
\text { issue of OSTBCs (unable to provide full-rate codes for }>2 \text { transmit antennas) can be overcome } \\
\text { with NOSTBCs but results in enhanced exponential decoding complexity in exchange for higher } \\
\text { rates. It is important to evaluate the expense in terms of decoding complexity linked with } \\
\text { relinquished orthogonality. }\end{array}$ \\
\hline & $\mathrm{v}$. & $\begin{array}{l}\text { A transmission using STBC performs worse than system using beam-forming technique. Crucial } \\
\text { gap in the performance between the two methods can be seen, especially in highly correlated } \\
\text { channels. }\end{array}$ \\
\hline & vi. & $\begin{array}{l}\text { Downside of methods relying on complete CSI at transmitter is feasibility and requirement for a } \\
\text { feedback path delivering CSI from the receiver to transmitter. In practical condition, in order to } \\
\text { save bandwidth in the feedback path, feedback channel may deliver only partial CSI to } \\
\text { transmitter. Moreover due to practical restrictions, the number of feedback bits per code block }\end{array}$ \\
\hline
\end{tabular}




\begin{tabular}{|c|c|c|}
\hline & & has to be kept as small as possible from receiver to transmitter. \\
\hline \multirow[t]{5}{*}{ Reed Solomon } & i. & $\begin{array}{l}\text { If a data stream is not corrupted by error bursts or drop-outs but random single bit errors are } \\
\text { present, a Reed-Solomon code is usually a poor choice. More efficient codes are available for } \\
\text { this case. }\end{array}$ \\
\hline & ii. & $\begin{array}{l}\text { Decoding of Reed-Solomon codes is a complex problem. It gives bulky and very complicated } \\
\text { code. Developer should have thorough knowledge in many areas of higher mathematics. }\end{array}$ \\
\hline & iii. & Barlekamp algorithms used to build an error polynomial are hard to implement or use. \\
\hline & iv. & The error probability for RS code shows poor BER performance for lower SNR. \\
\hline & $\mathrm{v}$. & $\begin{array}{l}\text { Other drawbacks of RS include delay penalty, enlarged bandwidth and practical implementation } \\
\text { obstacles. }\end{array}$ \\
\hline \multirow[t]{6}{*}{ Convolution } & i. & $\begin{array}{l}\text { In Hard and Soft decision decoding, the selection of quantization levels is crucial because of its } \\
\text { notable effect on the link performance. }\end{array}$ \\
\hline & ii. & $\begin{array}{l}\text { Higher numbers of parity bits demand proportionally higher amount of bandwidth for coding } \\
\text { overhead. }\end{array}$ \\
\hline & iii. & $\begin{array}{l}\text { In ML decoding, N-bit transmit sequence results in } 2^{\mathrm{N}} \text { possibilities making it highly unadaptable } \\
\text { to simply go through in sequence because of the sheer number. }\end{array}$ \\
\hline & iv. & $\begin{array}{l}\text { Due to parameters like finite trace-back length, branch and path metrics and input signal } \\
\text { quantization restricts physical implementation of a viterbi decoder to yield an exact ML stream. }\end{array}$ \\
\hline & $\mathrm{v}$. & $\begin{array}{l}\text { Viterbi algorithm suffers from path memory organization, decision memory reading techniques, } \\
\text { and the clocking mechanism. }\end{array}$ \\
\hline & vi. & $\begin{array}{l}\text { For high-speed applications, implementation of high-coding gain Viterbi decoders is } \\
\text { challenging due to the massive hardware size, larger power consumption and higher cost. }\end{array}$ \\
\hline \multirow[t]{5}{*}{ Turbo } & i. & $\begin{array}{l}\text { The major challenge with turbo code is the characteristic delay linked with the interleaver as } \\
\text { well as with the iterative decoding algorithm. }\end{array}$ \\
\hline & ii. & $\begin{array}{l}\text { The practical performance of turbo codes resides in the availability of a simple sub-optimal } \\
\text { iterative decoding. Though Iterative SOVA (Soft Output Viterbi Algorithm), output approaches } \\
\text { (ML) decoding performance bound with increased iterations. However, question of convergence } \\
\text { is still waiting for a concrete answer. }\end{array}$ \\
\hline & iii. & $\begin{array}{l}\text { A modified version of Viterbi algorithm, called SOVA used in decoding in turbo codes has } \\
\text { twice the complexity of Viterbi algorithm. }\end{array}$ \\
\hline & iv. & $\begin{array}{l}\text { The two main challenging aspects in interleaver design are the interleaver size and the } \\
\text { interleaver map. Turbo Decoder interleaver plays significant role in the trade-off between } \\
\text { performance and time (delay). }\end{array}$ \\
\hline & v. & $\begin{array}{l}\text { Problem with puncturing in codes is larger error events at high rates and higher distances. The } \\
\text { decision depth of such codes is quiet longer. Puncturing is a trade-off between rate and } \\
\text { performance. }\end{array}$ \\
\hline
\end{tabular}

\section{OPEN ISSUES AND FUTURE SCOPE}

Assorted gaps are still to be filled in coding techniques. Individual codes in their respective implications have positive and negative facets. RS codes are preferable for applications dealing with collective errors but they suffer from mathematical hitches and increased bandwidth problem which is yet to be solved. Immense performance gains in multipath fading wireless links can be annexed with Space-Time Coding (STC) techniques barring different factors like orthogonality, coding gain, CSI etc. that limit its efficiencies. No doubt LDPC codes are gaining attention but their flaws of higher architectural complications and lower resilience are still present. Talking about turbo coding the exact approach of information proceeding up-to next decoder and next iteration level is a matter of research. Exploration on better design layouts as well as link interleaver framing for code performance is required. Turbo coding has evolved as the most vibrant research area in wireless communications. These codes have potential to refine the user experience. There is further a scope of innovative changes in the coding techniques that will lead to their simplifications. Coding has ability to entirely transform mobile ecosystem by improving various efficiencies. There are some other codes such as concatenated codes and trellis coded modulation (TCM) codes having incredible flair. These are expected to surpass other codes in future. These codes open further opportunities for researchers to work upon.

\section{CONCLUSION}

There exist prominent tradeoffs for every code that have to be weighed against the application they are being applied for. This paper investigated convolution Codes, RS codes, LDPC, STBC and turbo codes, in the areas of both implementation and performance. A single FEC code with an optimum 
outputs for every application cannot be expected. FEC codes which are more effectual at resisting transmission errors have resulting expenses equally high in terms of hardware implementation and more complicated their encoding/decoding process becomes. However, all codes are being worked upon to overcome their gaps but still overall it can be said that Turbo codes outshine other codes when used for wireless communications. Vital fact is that shortcomings of turbo coding can be easily fulfilled using not too much complex alternatives. For example, using simply early termination, decoder can come out of loop before going through a set number of iterations reducing associated delay. With moderate decoding complexity and pretty good coding gains, turbo codes offer near optimum performance. In future, other codes are expected to vanquish their inadequacies making a smoother road to reach Shannon Limit.

\section{REFERENCES}

[1] Prabhakar Telagarapu, G.B.S.R.Naidu, K.Chiranjeevi (2011). "Analysis of Coding Techniques in WiMAX" International Journal of Computer Applications (0975 8887) Volume 22- No.3, May 2011.

[2] Priyanka Shrivastava, Uday Pratap Singh (2013). "Error Detection and Correction Using Reed Solomon Codes". Volume 3, Issue 8, August 2013 International Journal of Advanced Research in Computer Science and Software Engineering.

[3] M. A. M. El-Bendary, H. Kasban and M. A. R. ElTokhy(2014). "Interleaved Reed-Solomon Codes With Code Rate Switching over Wireless Communications Channels". 2nd International Conference on Advanced in Computer, Eletrical and Electronics Engineering (ICACEEE 2014) Co-jointed with 2nd International Conference on Computing, E-Learning and Emerging Technologies (ICCEET 2014) - Paris, France on July 8 9,2014 .

[4] Nazia Parveen, D.S. Venkateswarlu (2012). "Implementation of Space Time Block Coding using 2transmit and 2receive antennas".International Journal of Emerging Technology and Advanced Engineering, ISSN 2250-2459, Volume 2, Issue 10, October 2012.

[5] Khushbu Sethi, Vineet Sharma (2013). "Performance Evolution of Different Space Time Block Codes with Linear Receiver". International Journal of Engineering Research and Applications (IJERA) in 2013.

[6] Gunjan Manik, Alka Kalra, Sanjeev Kalra (2012). "Performance Analysis of STBC- OFDM System Under Multipath Fading Channel". International Journal of Soft Computing and Engineering (IJSCE) ISSN:2231-2307, Volume-1, Issue-6, January 2012.
[7] Sanjeev Kumar, Ragini Gupta (2011). "Performance Comparison of Different Forward Error Correction Coding Techniques for Wireless Communication Systems". IJCST Vol. 2, Issue 3, September 2011.

[8] Mr.Vishal G. Jadhao, Prof.Prafulla D. Gawande (2012) "Performance Analysis of Linear Block Code, Convolution code and Concatenated code to Study Their Comparative Effectiveness". IOSR Journal of Electrical and Electronics Engineering (IOSRJEEE) Volume 1, Issue 1 (May-June 2012).

[9] Syed MD. Asif, Abdullah - Al - Maruf, S. M. Anisul Islam, Amitavo Tikader, MD. Abdul Alim. "Comparison of ber between uncoded signal and coded signal (using convolution code) over slow rayleigh fading channel". Journal of Theoretical and Applied Information Technology, Jan 2010.

[10] Bhasker Gupta and Davinder S. Saini (2013). "Performance of Concatenated Optimized Irregular LDPC Code with Alamouti Coded MIMO-OFDM Systems". WSEAS TRANSACTIONS on COMMUNICATIONS Issue 5, Volume 12, May 2013.

[11] Rutuja Shedsale, Nisha Sarwade (2012). "A REVIEW OF CONSTRUCTION METHODS FOR REGULAR LDPC CODES". Indian Journal of Computer Science and Engineering (IJCSE) Vol. 3 No. 2 Apr-May 2012.

[12] Alaa Eldin.S. Hassan, Moawad Dessouky, Atef Abou Elazm and Mona Shokair (2012). "Evaluation of Complexity Versus Performance for Turbo Code and LDPC Under Different Code Rates". SPACOMM 2012: The Fourth International Conference on Advances in Satellite and Space Communications.

[13] Ahmad Hasan Khan, Dr K C Roy (2013). "Comparison of Turbo Codes and Low Density Parity Check Codes". IOSR Journal of Electronics and Communication Engineering (IOSR-JECE) Volume 6, Issue 6 (Jul. - Aug. 2013).

[14] Patel Sneha Bhanubhai, Grace Mary Shajan, Dalal D. Upena (2012). "Performance of Turbo Encoder and Turbo Decoder for LTE" International Journal of Engineering and Innovative Technology (IJEIT) Volume 2 , Issue 6

[15] Nimbalker Ajit, Blankenship Yufei, Classon Brian (2008). "ARP and QPP Interleavers for LTE Turbo Coding" in WCNC.

[16] Jagdish D. Kene and Dr. Kishor D. Kulat (2013). "Iterative Decoding Termination Schemes for Turbo Code Performance Optimization In Mobile Wi-Max Environment". IJCSI International Journal of Computer Science Issues, Vol. 10, Issue 4, No 1, July2013. 